\title{
Pre-Existing Microbiome Signature in a SARS-CoV-2 Discordant Family
}

\author{
Sabine Hazan \\ Progenabiome, Ventura Clinical Trials, Ventura, CA, USA
}

\section{Keywords}

Microbiome signature $\cdot$ COVID-19 $\cdot$ SARS-CoV-2 $\cdot$ Bifidobacterium

\begin{abstract}
Our index patient is a 19-year-old man with Crohn's disease. After developing symptoms consistent with COVID-19, he, his 62-year-old father, and 14-year-old sister tested positive for SARS-CoV-2 in May 2020. Despite a shared household, his 50-year-old mother with a history of asthma and his healthy brother and sister-in-law (a married couple) remained negative. The index patient and his mother had undergone microbiome analysis in May 2019, following his brother and his sister-in-law in November 2020. We observed significant differences between the fecal microbiota of the SARS-CoV-2-positive son and those of his healthy family. There were differences in the bacterial phylum, class, order, family, and genus level with the increased relative abundance of Bacteroidetes and reductions or deletions in bacterial diversity, particularly of the Bifidobacterium family. This unique study may signal a new exploratory avenue for the prevention or treatment of SARS-CoV-2 infections.
\end{abstract}

(c) 2022 The Author(s).

Published by S. Karger AG, Basel

\section{Introduction}

Little is known of the molecular mechanisms or genetic predispositions that may underpin the hyperinflammatory response during or after acute infection [1]. Mounting scientific evidence confirms that infections, including SARS-CoV-2, can provoke a significant loss of microbial diversity in the gut and that this loss can suppress the immunologic response [2, 3]. Two important microbiota commensals, Bifidobacteria and Lactobacillus [4], are significantly reduced in COVID-19 patients [5]. SARS-CoV-2 may also alter cytokine levels that increase the severity of COVID-19. Enhancing gastrointestinal microbiome diversity might be helpful for lowering SARS-CoV-2 infection risk or COVID-19 severity [6, 7]. 
We describe a household comprising an index SARS-CoV-2-positive patient with a history of Crohn's, who along with his mother, had microbiome analysis in 2019. His brother and sister-in-law who shared the same household, underwent microbiome analysis in late 2020. The clinical characteristics and microbiome analysis for the four family members are compared. The mother, brother, and sister-in-law tested negative for SARS-CoV-2 by both PCR and antibody tests, and the son tested positive by both antibody and PCR tests. The household also included two other family members - the patient's father and sister; both were SARS-CoV-2-positive during the same timeframe, but neither had a microbiome analysis before or during the pandemic.

\section{Case Report/Case Presentation}

The household included the 19-year-old index patient who had Crohn's disease, his 50 -year-old mother with a history of asthma and no medication prior to her visit, and his 28-year-old brother, 26-year-old sister-in-law, 62-year-old father, and 14-year-old sister. The mother and son visited the Clinic in May 2019 to consult regarding the son's Crohn's disease. At the time, he was using mesalamine delayed release $1.2 \mathrm{~g}$ tablets. Following the consultation in May 2019, the patient, and his mother both underwent microbiome analysis.

In May 2020, the index patient developed fever $\left(102^{\circ} \mathrm{C}\right)$, sore throat, rhinorrhea, productive cough, and diarrhea, but no dyspnea. He tested positive for SARS-CoV-2 by PCR. His mesalamine delayed release was discontinued, and he was treated with 5 days of hydroxychloroquine $200 \mathrm{mg}$ po BID, vitamin C 3,000 mg po qd, and zinc $50 \mathrm{mg}$ po qd and 10 days of vitamin D 3,000 IU po qd.

The patient's 62-year-old father and 14-year-old sister tested positive for SARS-CoV-2 by PCR within 2 days of the son's positive test. There was no sample collected at the sickness time due to the SARS-CoV-2 safety concerns to collect feces by both the father and daughter. The mother was the sole caregiver for the sick family members and shared a bed and bathroom with her ill husband. The mother's PCR collected on the same day as those for the rest of the family was negative. At the same period, he infected the remainder of her family with SARSCoV-2. The healthy brother and sister-in-law had no underlying conditions, were on no medications, and tested negative for SARS-CoV-2 on the same day as all family members were tested. The family has never used any pro- or prebiotics, they shared the same diet, and all had body mass indices $<26$. The mother, brother, and sister-in-law have remained well since May 2020.

We conducted a microbiome analysis on a stool from four family members. We compared interindividual differences between the index patient and his mother, brother, and sister-inlaw in phylum, class, order, family, and genus. There was no sample collected for father and daughter at the sickness time due to the SARS-CoV-2 safety concerns for feces collections by them. We have a continuous validation system for microbiome analysis. Therefore, all microbiome analysis assays through the clinical study were reproducible through the study period. Additionally, there were not any changes in the family diet and medicine through the period.

We obtained written informed consent from all subjects before initiating the study procedure. DNA was extracted from the stools of the index patient and mother in May 2019 and for the brother and sister-in-law in November 2020. DNA was then quantitated and normalized for downstream library fabrication utilizing Hazan-Papoutsis shotgun methodology [8]. Prepared and indexed libraries were subsequently pooled and sequenced on the Illumina NextSeq 550 System. Sample FASTQ files were analyzed with a computational tool profiling the microbial communities from metagenomic sequencing data with a species-level resolution. Finally, we compared the microbiome of the SARS-CoV-2-positive index patient

\section{Karger'}


Hazan: Case Study of Microbiome in SARS-CoV-2

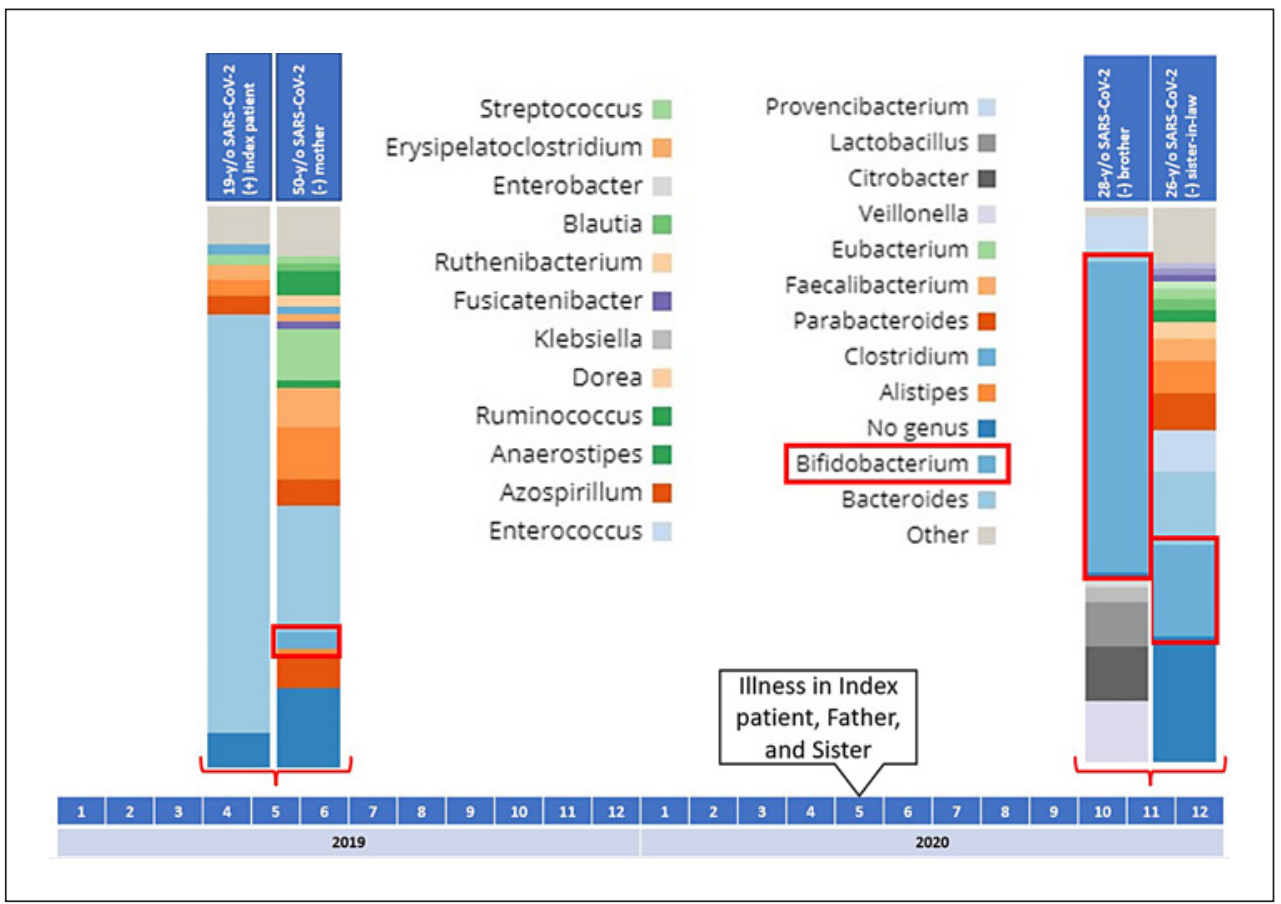

Fig. 1. The relative abundance of various genera including Bifidobacterium in the SCV+19-year-old index patient and the SCV- 50-year-old mother, 28-year-old brother, and 26-year-old sister-in-law by month of collection. SCV+, SARS-CoV-2-positive; SCV-, SARS-CoV-2-negative.

with those of his SARS-CoV-2-negative mother, brother, and sister-in-law in the same household, using OneCodex Software using both quick compare, custom plot, and OneCodex Jupyter notebook using Python for Shannon and Simpson Index, Alpha and beta diversity, and Chao analysis.

In total, four stool samples were sequenced for this study. We compared gut microbiota composition and relative abundance in the stool samples of the SARS-CoV-2-positive patient and his SARS-CoV-2-negative family members to assess whether their gut microbiota composition differed. We hypothesized that the patient and well household members might have different microbiota signatures at the family and genus levels.

Figure 1 depicts the relative abundance at the genus level, including Bifidobacterium genus in the SARS-CoV-2-positive (+) index patient and the SARS-CoV-2-negative (-) mother, brother, and sister-in-law by the month of collection. The initial difference between the microbiome of mother and son prior the COVID-19 period is possibly due to the mother's Crohn's disease involvement. Figure 2 and Table 1 show relative abundance reductions and expansions at the phylum level and the same for class, order, family, and genus for the microbiome of the SARS-CoV-2-positive son compared to those of his SARS-CoV-2-negative mother, brother, and sister-in-law. Reductions were observed at the phylum level for Actinobacteria, class level for Actinobacteria, order level for Bifidobacteriales, family level for Bifidobacteriaceae and Ruminococcaceae, and at the genus level for Bifidobacterium. Expansions were observed in the microbiome of the SARS-CoV-2-positive index patient compared to his negative household members at the phylum level for Bacteroidetes, class level for Bacteroidia, order level for Bacteroidales, and family level for Bacteroidaceae, and genus level for Bacteroides.

We calculated alpha diversity based on the gene profiles using the Shannon and Simpson index. The Simpson microbial diversity (alpha diversity) was significantly decreased in 
Hazan: Case Study of Microbiome in SARS-CoV-2

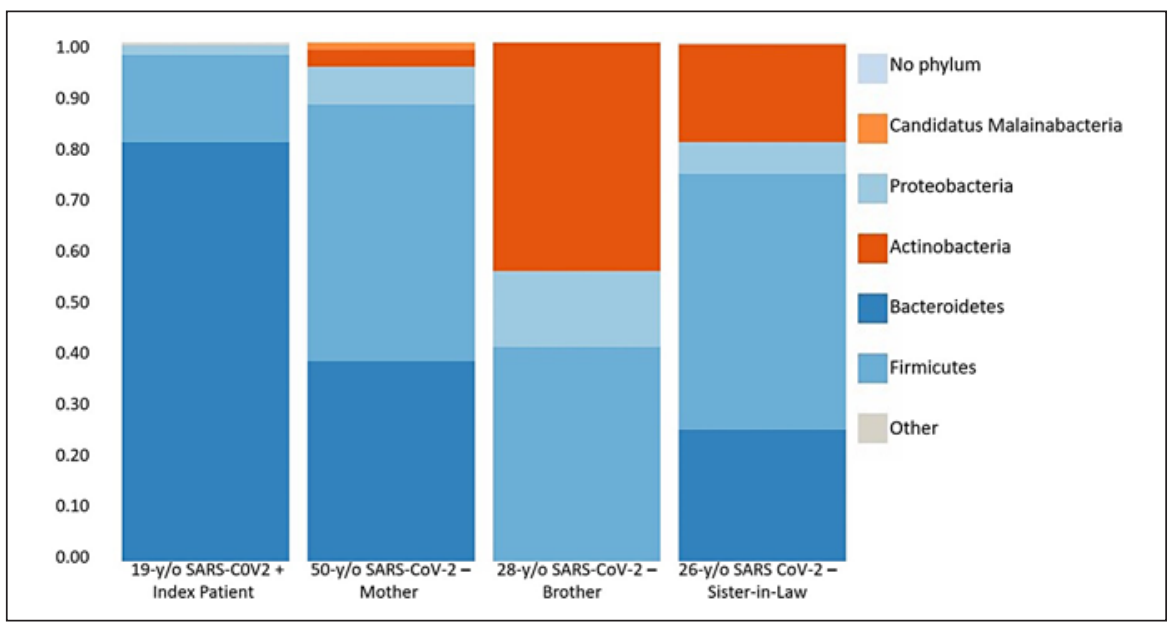

Fig. 2. Phylum-level relative abundance.

Table 1. Relative abundance of index case, mother, brother, and sister-in-law at phylum, class, order, family, and genus levels for Bifidobacterium and Bacteroides

\begin{tabular}{|c|c|c|c|c|c|}
\hline \multirow[t]{2}{*}{ Level } & \multirow[t]{2}{*}{ Group } & \multicolumn{4}{|c|}{ Relative abundance, \% } \\
\hline & & index case & mother & brother & sister-in-law \\
\hline \multicolumn{6}{|l|}{ Reductions } \\
\hline Phylum & Actinobacteria & 0.4 & 3.0 & 43.0 & 18.7 \\
\hline Class & Actinobacteria & 0.0 & 3.0 & 43.0 & 16.6 \\
\hline Order & Bifidobacteriales & 0.1 & 3.0 & 43.0 & 16.6 \\
\hline Family & $\begin{array}{l}\text { Bifidobacteriaceae } \\
\text { Ruminococcaceae }\end{array}$ & $\begin{array}{l}0.0 \\
4.0\end{array}$ & $\begin{array}{l}3.0 \\
14.4\end{array}$ & $\begin{array}{l}44.0 \\
0.0\end{array}$ & $\begin{array}{l}16.6 \\
17.0\end{array}$ \\
\hline Genus & Bifidobacterium & 0.0 & 3.0 & 44.0 & 16.6 \\
\hline \multicolumn{6}{|l|}{ Expansions } \\
\hline Phylum & Bacteroidetes & 81.0 & 38.6 & 0.0 & 25.5 \\
\hline Class & Bacteroidia & 81.0 & 38.6 & 0.0 & 25.4 \\
\hline Order & Bacteroidales & 81.0 & 38.6 & 0.0 & 25.4 \\
\hline Family & Bacteroidaceae & 74.5 & 22.5 & 0.0 & 13.0 \\
\hline Genus & Bacteroides & 74.5 & 22.5 & 0.0 & 13.0 \\
\hline
\end{tabular}

Relative abundance expansions and reductions in a SARS-CoV-2 discordant family.

SARS-CoV-2-positive patient compared to his negative mother, brother, and sister-in-law accordingly $(0.66,0.96,0.96$ and, $0.85 p<0.00001$ for Simpson diversity). The Shannon diversity index was $(2.94,5.52,5.53,3.65)$ accordingly.

\section{Discussion/Conclusion}

We are unaware of any other microbiome analyses for SARS-CoV-2-positive and negative family members for which studies in both a positive and negative family member preceded the COVID-19 pandemic. The mother was the sole caregiver for all the sick family members 
and shared a bed and bathroom with her SARS-CoV-2-positive husband. That the mother, brother, and sister-in-law were PCR-negative and remained asymptomatic despite living with three positive family members suggests that considerable exposure, and second, that some of the family members were less susceptible to infection than others. The composition and relative abundance of various phyla, classes, orders, families, and genera in the gut microbiota of the family members who remained well may have played a role in modulating their immune responses.

Gut microbiota influence and modulate host immune responses [9]. For example, crosstalk between the liver and the intestine is essential for natural immune surveillance, adaptive immune response regulation, and nutrient metabolism, and elimination of toxic bacterial metabolites [10]. Additionally, in HIV patients, the altered gut microbiome increases disease susceptibility [11].

In our case, a year before he became infected, the SARS-CoV-2-positive index patient had significantly less beneficial Bifidobacterium commensals and significantly more Bacteroides than his uninfected mother. He also had fewer beneficial and more Bacteroides than his uninfected brother and sister-in-law, who volunteered to be tested later during the COVID-19 pandemic.

Several authors have hypothesized that the microbiome might influence susceptibility to SARS-CoV-2 infection or COVID-19 severity [12-15], and Ahlawat has described immunological coordination between the gut and lung in SARS-CoV-2 infections [16]. A few groups have assessed the microbiomes of patients hospitalized for COVID-19. Zuo et al. [17] observed a fungal dysbiosis in patients hospitalized for COVID-19, with increases in Candida albicans, Candida aureus, and Aspergillus flavus; his group observed a similar dysbiosis in the microbiome of patients with community-acquired pneumonia but not in those of healthy controls. Zuo et al. [17] And Yeoh et al. [18] described the microbiomes of medicated and nonmedicated COVID-19 patients collected during hospitalization as significantly different from those of uninfected individuals $(p<0.01)$. Gut commensals with known immunomodulatory potential such as Faecalibacterium prausnitzii, Eubacterium rectale, and Bifidobacteria were underrepresented in the microbiome COVID-19 patients compared to the microbiome of uninfected individual [18].

An aggressive inflammatory response is a major pathophysiological characteristic of SARSCoV-2 infections [18]. Yeoh's findings of reductions in relative abundance of Faecalibacterium and Eubacterium rectale are of interest; both species may use dietary fiber to increase butyrate ratio/levels [19] which, in turn, can down-regulate inflammation [20]. However, we saw relative abundance reductions in neither of these species. Like Yeoh et al. [18], who compared hospitalized COVID-19 patients and controls, we observed a significantly lower relative abundance of important Bifidobacterium commensals in the son's microbiome compared to those of his mother, brother, and sister-in-law. However, we also observed expansions in the relative abundance of Bacteroidetes. Setoyama et al. [21] has suggested that the severity of gut inflammation in ulcerative colitis may be linked to Bacteroides vulgatus, and that this inflammation might be repressed by Bifidobacterium. Bifidobacteria are Gram-positive, anaerobic, saccharolytic Actinobacteria that fermentatively degrade complex carbohydrates and produce acetate and lactate, which are, in turn, used by butyrateand propionate producing colon bacteria [22]. These two primary short-chain fatty acids butyrate and propionate - have significant anti-inflammatory effects. Bifidobacteria are particularly abundant in infants and decline steadily with age. Breastfed infants have higher levels of bifidobacterial than formula-fed infants, and the levels decrease after breastfeeding ends. Various Bifidobacteria spp. have been shown to induce dendritic cell maturation, T-cell polarization (toward a Th1 response), production of the modulatory cytokine IL10, and decreases in TNFa and INFg production, and subcellular fractions of Bifidobacteria

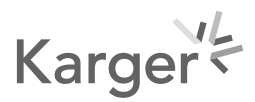


have been identified as effectors of CD8C T-cell activation [23]. We hypothesize that microbiome alterations at the phylum level may have increased the son's susceptibility to SARSCoV-2 compared to his household members, who were negative and remained asymptomatic, mainly because there is increasing evidence of gut microbiota dysbiosis in COVID-19 patients despite respiratory etiology of the disease [24-26]. If this were confirmed in additional studies, a baseline microbiome analysis might predict SARS-CoV-2 susceptibility and buy time for dietary interventions with pre- or probiotics to improve resistance. This hypothesis is supported by Kim et al. [27], who recently stated that an altered and leaky gut microbiota might allow SARS-CoV-2 into the circulatory system and result in multiorgan complications.

Limitations of our data are as follows. We report on a single household, and only one of the three persons who became ill had a prior microbiome analysis. Although everyone was tested at one point in time and three of the household members - the index patient's mother, brother, and sister-in-law - remained healthy, any of the three might have developed asymptomatic SARS-CoV-2 infections later. It would have been desirable to repeat the microbiome analysis for the son after his illness and note any post-infection alterations and perform antibody tests in the PCR-negative asymptomatic family members to look for evidence of infection.

\section{Conclusion}

We suspect that a baseline microbiome analysis might predict SARS-CoV-2 susceptibility and that a "susceptible" signature might be remediable. Although our report of this SARS-CoV-2 discordant household only includes data for four persons, dietary and social/ socioeconomic factors were constant across family members. Given the high attack rates within families, additional studies of discordant family units would be instrumental. An epidemiological study of families with members who have similar pre-pandemic microbiome "signatures" might help establish whether decreases in Bifidobacterium or increases in Bacteroidetes could predict SARS-CoV-2 infection or COVID-19 severity risk.

\section{Acknowledgement}

The authors thank Dr. Mehrdad Tajkarimi, chairman of the board, Arya Technologies LLC (Greensboro, NC, USA).

\section{Statement of Ethics}

This study protocol was reviewed and approved by Advarra IRB as Pro00043762. Written informed consent was obtained from the patient for publication of the details of their medical case and any accompanying images.

\section{Conflict of Interest Statement}

Potential conflicts of interest: Dr. Sabine Hazan MD owns ProgenaBiome and Ventura Clinical Trials, which may constitute a competing interest.

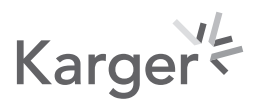


Hazan: Case Study of Microbiome in SARS-CoV-2

\section{Funding Sources}

This research received no external funding.

\section{Author Contributions}

Dr. Sabine Hazan is the sole clinician, investigator, and author of this case study.

\section{Data Availability Statement}

All data generated or analyzed during this study are included in this article. Further inquiries can be directed to the corresponding author.

\section{References}

1 Galeotti C, Bayry J. Autoimmune and inflammatory diseases following COVID-19. Nat Rev Rheumatol. 2020 Aug;16(8):413-4.

2 Huggins MA, Sjaastad FV, Pierson M, Kucaba TA, Swanson W, Staley C, et al. Microbial exposure enhances immunity to pathogens recognized by TLR2 but increases susceptibility to cytokine storm through TLR4 sensitization. Cell Rep. 2019 Aug 13;28(7):1729-43.e5.

3 Tay MZ, Poh CM, Rénia L, MacAry PA, Ng LFP. The trinity of COVID-19: immunity, inflammation and intervention. Nat Rev Immunol. 2020 Jun;20(6):363-74.

4 Stavropoulou E, Bezirtzoglou E. Probiotics in medicine: a long debate. Front Immunol. 2020;11:2192.

5 Tiwari SK, Dicks LMT, Popov IV, Karaseva A, Ermakov AM, Suvorov A, et al. Probiotics at war against viruses: what is missing from the picture? Front Microbiol. 2020;11:1877.

$6 \mathrm{Gu}$ S, Chen Y, Wu Z, Chen Y, Gao H, Lv L, et al. Alterations of the gut microbiota in patients with coronavirus disease 2019 or H1N1 influenza. Clin Infect Dis. 2020 Dec 17;71(10):2669-78.

7 Santacroce L, Charitos IA, Ballini A, Inchingolo F, Luperto P, De Nitto E, et al. The human respiratory system and its microbiome at a glimpse. Biology. 2020 Oct 1;9(10):318.

8 Hazan S, Spradling-Reeves KD, Papoutsis A, Walker SJ. Shotgun metagenomic sequencing identifies dysbiosis in triplet sibling with gastrointestinal symptoms and ASD. Children. 2020 Nov 25;7(12):255.

9 Bui A, Choi Y, Frankel AE, Koh AY. Unbiased microbiome and metabolomic profiling of fecal samples from patients with melanoma. Methods Mol Biol. 2021;2265:461-74.

10 Shao JW, Ge TT, Chen SZ, Wang G, Yang Q, Huang CH, et al. Role of bile acids in liver diseases mediated by the gut microbiome. World J Gastroenterol. 2021 Jun 14;27(22):3010-21.

11 Abange WB, Martin C, Nanfack AJ, Yatchou LG, Nusbacher N, Nguedia CA, et al. Alteration of the gut fecal microbiome in children living with HIV on antiretroviral therapy in Yaounde, Cameroon. Sci Rep. 2021 Apr 7;11(1): 7666.

12 He LH, Ren LF, Li JF, Wu YN, Li X, Zhang L. Intestinal flora as a potential strategy to fight SARS-CoV-2 infection. Front Microbiol. 2020;11:1388.

13 Terruzzi I, Senesi P. Does intestinal dysbiosis contribute to an aberrant inflammatory response to severe acute respiratory syndrome coronavirus 2 in frail patients? Nutrition. 2020 Nov-Dec;79-80:110996.

14 Walton GE, Gibson GR, Hunter KA. Mechanisms linking the human gut microbiome to prophylactic and treatment strategies for COVID-19. Br J Nutr. 2021;126(2):219-27.

15 Chattopadhyay I, Shankar EM. SARS-CoV-2-indigenous microbiota nexus: does gut microbiota contribute to inflammation and disease severity in COVID-19? Front Cell Infect Microbiol. 2021;11:590874.

16 Ahlawat S, Asha, Sharma KK. Immunological co-ordination between gut and lungs in SARS-CoV-2 infection. Virus Res. 2020;286:198103.

17 Zuo T, Zhan H, Zhang F, Liu Q, Tso EYK, Lui GCY, et al. Alterations in fecal fungal microbiome of patients with COVID-19 during time of hospitalization until discharge. Gastroenterology. 2020 0ct;159(4):1302-10.e5.

18 Yeoh YK, Zuo T, Lui GC, Zhang F, Liu Q, Li AY, et al. Gut microbiota composition reflects disease severity and dysfunctional immune responses in patients with COVID-19. Gut. 2021 Apr;70(4):698-706.

19 Nilsen M, Madelen Saunders C, Leena Angell I, Arntzen MO, Lodrup Carlsen KC, Carlsen KH, et al. Butyrate levels in the transition from an infant- to an adult-like gut microbiota correlate with bacterial networks associated with Eubacterium rectale and Ruminococcus gnavus. Genes. 2020 Oct 22;11(11):1245.

20 Chen J, Vitetta L. Inflammation-modulating effect of butyrate in the prevention of colon cancer by dietary fiber. Clin Colorectal Cancer. 2018 Sep;17(3):e541-4. 
Hazan: Case Study of Microbiome in SARS-CoV-2

21 Setoyama H, Imaoka A, Ishikawa H, Umesaki Y. Prevention of gut inflammation by Bifidobacterium in dextran sulfate-treated gnotobiotic mice associated with Bacteroides strains isolated from ulcerative colitis patients. Microbes Infect. 2003 Feb;5(2):115-22.

22 De Vuyst L, Moens F, Selak M, Rivière A, Leroy F. Summer meeting 2013: growth and physiology of bifidobacteria. J Appl Microbiol. 2014 Mar;116(3):477-91.

23 Ruiz L, Delgado S, Ruas-Madiedo P, Sánchez B, Margolles A. Bifidobacteria and their molecular communication with the immune system. Front Microbiol. 2017;8:2345.

24 Ferreira C, Viana SD, Reis F. Gut microbiota dysbiosis-immune hyperresponse-inflammation triad in Coronavirus Disease 2019 (COVID-19): impact of pharmacological and nutraceutical approaches. Microorganisms. 2020 Oct 1;8(10):1514.

25 Zhou F, Yu T, Du R, Fan G, Liu Y, Liu Z, et al. Clinical course and risk factors for mortality of adult inpatients with COVID-19 in Wuhan, China: a retrospective cohort study. Lancet. 2020 Mar 28;395(10229):1054-62.

26 Yeoh YK, Zuo T, Lui GC, Zhang F, Liu Q, Li AY, et al. Gut microbiota composition reflects disease severity and dysfunctional immune responses in patients with COVID-19. Gut. 2021 Apr;70(4):698-706.

27 Kim HS. Do an altered gut microbiota and an associated leaky gut affect COVID-19 severity? mBio. 2021 Jan 12;12(1). 